\title{
Phenotypic plasticity and similarity of DNA among genotypes of an annual plant
}

\author{
M. JASIEŃSKI*†, FRANCISCO JOSÉ AYALA † \& \&. A. BAZZAZ† \\ †Department of Organismic and Evolutionary Biology, Harvard University, 16 Divinity Avenue, Cambridge, MA \\ 02138 and $\ddagger 208$ Mueller Laboratories, Institute of Molecular Evolutionary Genetics, Pennsylvania State University, \\ University Park, PA 16802, U.S.A.
}

\begin{abstract}
When measured directly, rather than inferred from pedigree analyses, the relationship between similarity in phenotype and similarity in DNA sequence was detectable at the level of members of a single population and strongly depended on the environmental context. Genetic divergence among 27 co-occurring genotypes of Abutilon theophrasti, a common annual plant, was less than 5 per cent as revealed by RAPD-PCR analysis based on over 400 bands per genotype. Nevertheless, within this narrow range, there was a positive correlation between genetic similarity and similarity in the performance of genotypes on temperature and moisture gradients, suggesting that plasticity itself has a genetic basis. No relationship was detected, however, when the phenotypic plasticity was expressed in response to gradients of light intensity or soil fertilization, indicating a weaker genetic basis, or suggesting possible involvement of a few genes of major effect.
\end{abstract}

Keywords: genetic similarity, genotype, niche overlap, norm of reaction, phenotypic plasticity, RAPD-PCR.

\section{Introduction}

The existence of a congruence between genomic divergence and phenotypic difference is a fundamental assumption of evolutionary genetics (Damerval et al., 1987; Scharloo, 1991; Villani et al., 1992). For example, the decline of phenotypic similarity with decreasing relatedness among competing related conspecifics is postulated as one of the critical factors in the evolution of sexual reproduction and social behaviour. The extent of a congruence may depend, however, on the specific characteristics of the loci involved in determining focal phenotypic traits of the studied organisms or on the context in which such loci are functioning, i.e. linkage patterns (Scharloo, 1991; Lander \& Schork, 1994; Moreno, 1994). Although great morphological similarity of sibling species is frequently associated with high genetic similarity (Lewontin, 1974), one should not expect overall genetic similarity to be correlated with morphological similarity at the phylogenetic level, if morphological evolution involves changes in only a few regulatory sequences. The type of genomic vari-

*Correspondence. E-mail: mjasiens@oeb.harvard.edu ation that is reflected in phenotypic variation sets the limit to the efficiency of natural selection and determines the boundary between the domains of neutral and adaptive evolution. If the trait in question is a compound characteristic, such as phenotypic plasticity, which describes an overall responsiveness of the organism rather than a phenotypic detail, precise mapping and detecting a gene dosage-phenotype relationship may not be feasible. In this study, we have therefore used a simple statistical approach to address the type of relationship between the genotypic and the phenotypic space.

Phenotypic plasticity is the ability of a single genotype to produce an array of phenotypes, depending on the environmental context (Schlichting \& Levin, 1984; Via et al., 1995). Within species, variation in the expression of phenotypic plasticity has been documented among genotypes from a single population (e.g. Macdonald \& Chinnappa, 1989) or among populations of the same species (e.g. Taylor \& Aarssen, 1988; Sultan \& Bazzaz, 1993; but see Counts, 1993). Schlichting \& Levin (1984) postulated that more closely phylogenetically related taxa should vary less in their patterns of plasticity. Here we ask whether genetic similarity correlates with 
ecological similarity at the intraspecific level, among genotypes from the same population.

Attempts to quantify genetic similarity at the DNA level among intraspecific categories (ecotypes, crop varieties) have only recently been facilitated by technological advances, such as random amplification of polymorphic DNA (RAPD) or restriction fragment length polymorphism (RFLP) analyses. It is therefore not surprising that there have been very few studies of the congruence between phenotypic and genotypic space that would rely on the direct measurements of the phenotypic and genetic similarity. Damerval et al. (1987) found a positive correlation among five maize lines between the morphological distance based on 14 heritable traits and an index of genetic distance based on variation in the intensity of electrophoretic spots. At the interpopulation level, genetic and morphophysiological patterns of variation were significantly congruent among Turkish populations of Castanea sativa (Villani et al., 1992). There was some concordance in genetic similarity (from isozyme analysis) and similarity in phenotypic plasticity among populations of Zizania species (Counts, 1993), but the small number of populations precluded statistical tests of the relationship. Dendrograms based on 24 phenotypic characters only partially reflected similarity in DNA among inbred strains of Microseris elegans originating from nine populations (Bachmann \& Van Heusen, 1992), although an explicit statistical analysis of the congruence was lacking. Finally, population divergence in phenotypic plasticity was not correlated with that of isozyme profiles in the Stellaria longipes complex (Macdonald \& Chinnappa, 1989).

The evolutionary significance of phenotypic plasticity must be evaluated with explicit reference to quantitative and molecular genetics, as both genetic correlations (Via et al., 1995) and molecular biology of gene expression (Lander \& Schork, 1994; Mitchell-Olds, 1995) influence the rate and trajectory of evolution (Orr \& Coyne, 1992). However, the genetic underpinnings of phenotypic plasticity, especially the relative importance of genes of major vs. minor effect, are the subject of a recent controversy (Scheiner, 1993; Schlichting \& Pigliucci, 1995; Via et al., 1995).

Here, we attempt to infer the underlying genetic basis of phenotypic plasticity within genotypes by studying the pattern of the relationship between ecological and genetic similarity among genotypes. Correlation of phenotypic and genotypic differences has usually been quantified through traditional breeding methods and pedigree analysis. We have measured it directly by comparing patterns of similarity of genomes (as revealed by RAPDs analysis) with patterns of similarity in phenotypic plasticity, in a sample of wild genotypes of an annual plant grown under four broad environmental gradients.

\section{Materials and methods}

Ecology and phenotypic plasticity of Abutilon theophrasti (Malvaceae) has been studied extensively by F. A. Bazzaz and co-workers (Bazzaz et al., 1995; Bazzaz, 1996). In this study, phenotypic plasticity was assessed by examining the ecological performance of over 2800 plants of Abutilon in four separate experiments (on broad gradients of light, moisture, nutrient and temperature). Original genotypes were sampled as seeds from a natural population in an old-field in Illinois, and the resulting plants were cloned through a tissue culture technique of axillary bud enhancement. Clonally propagated genotypes were grown as individual plants in a controlledenvironment glasshouse.

\section{Quantification of plasticity}

The light gradient consisted of five levels of light intensity ( 5 per cent, 21 per cent, 33 per cent, 62 per cent and 100 per cent), obtained through the use of neutral shade cloth of different densities suspended above the growing plants, with six clonal members of each genotype per light level (a total of 600 plants from 20 genotypes). Five levels of soil moisture (10 per cent, 15 per cent, 20 per cent, 30 per cent and 40 per cent) were produced by an automatic watering system, with 6-9 replicates of each genotype at each moisture level (a total of 720 plants from 20 genotypes). The highest level of moisture corresponded to the field-capacity soil conditions. The nutrient gradient consisted of five levels of Peter's nitrogen-phosphorus-potassium fertilizer $(0$ per cent, 12.5 per cent, 25 per cent, 50 per cent and 100 per cent), corresponding to the addition of 0,62 , 125,250 and $500 \mathrm{mg}$ of nitrogen per plant. The experiment was set up in a randomized complete block design with 10 blocks and no replication of genotypes within blocks (a total of 1000 plants from 20 genotypes). A gradient of six levels of temperature $\left(15 / 12,20 / 16,25 / 20,30 / 24,35 / 28\right.$ and $40 / 32^{\circ} \mathrm{C}$, day/night) was established in 12 glass chambers, with two chambers per temperature level and six replicates per genotype at each temperature (a total of 576 plants from 16 genotypes). Eight genotypes (out of 27) were tested on all four gradients and analyses of their responses allow for comparisons of pheno- 
typic plasticity among environmental gradients, without the confounding effects of variation in the identity of genotypes.

\section{DNA analysis}

Random amplification of polymorphic DNA (RAPD) by the polymerase chain reaction (Hadrys et al., 1992) was used to measure genetic similarity among 27 genotypes of Abutilon theophrasti. RAPDPCR yields information about both coding and noncoding regions of the genome, and is especially suitable for the study of closely related genomes (Tinker et al., 1993; Mitchell-Olds, 1995). Total genomic DNA was extracted from young leaves of a single plant per genotype (grown from seeds produced after selfing by the original clones) by standard CTAB isolation methods. Twenty RAPD primers (OPA-1 to OPA-20; Operon Technologies, Alameda, CA, U.S.A.) were used in the analyses.

\section{Data analyses}

The index of genetic similarity $(F)$ was calculated for all pairs of genotypes using Nei \& Li's matching coefficient method:

$F=2 N_{j k} /\left(\mathrm{N}_{j}+N_{k}\right)$,

where $N_{j k}$ is the number of bands shared by both genotypes and $N_{j}$ and $N_{k}$ are the numbers of bands unique to genotypes $j$ and $k$, respectively ( $\mathrm{Nei}$, 1987). For each genotype and pairwise comparison, the values of $N_{j}, N_{k}$ and $N_{j k}$ were summed over the results from all primers. Overall similarity of ecological performance at each gradient (i.e. similarity in the phenotypic plasticity of total fruit biomass production) was quantified for each pair of genotypes, $j$ and $k$, using the Horn's index of resource overlap (Krebs, 1989), according to the formula:

$$
\begin{aligned}
R_{\mathrm{o}}= & {\left[\Sigma\left(p_{i j}+p_{i k}\right) \log \left(p_{i j}+p_{i k}\right)-\Sigma p_{i j} \log p_{i j}\right.} \\
& \left.-\Sigma p_{i k} \log p_{i k}\right] /(2 \log 2),
\end{aligned}
$$

where $p_{i j}$ and $p_{i k}$ are proportional responses of genotypes $j$ and $k$, respectively, attained in the $i$ th resource state. Horn's index has been shown to have smaller bias than other commonly used indices (Krebs, 1989). All similarity matrices were presented as dendrograms, using the UPGMA method of clustering, as implemented in the NTSYS-pc program (Rohlf, 1994). Goodness-of-fit of the dendrograms to the original matrices was evaluated by the cophenetic correlation coefficients, with 5000 randomizations for significance tests (Rohlf, 1994).
To examine the relationships between genetic and phenotypic similarities, matrices of phenotypic similarity obtained from each of the gradients were compared individually with the matrix of DNA similarity using a Mantel test (Manly, 1991). Probability levels are based on 20000 permutations, using the RT program (West, Inc., Cheyenne, WY, U.S.A.). Analyses were performed on all genotypes tested at each gradient and then on the subset of eight genotypes for which information existed from all four experiments.

\section{Results and discussion}

RAPD primers yielded on average 415 scoreable bands per genotype (range 409-429). The most divergent pair of genotypes differed in 30 bands (index of genetic similarity of 94.8 per cent), whereas the most similar pair differed in only two bands (similarity of 99.4 per cent) (Fig. 1). Variation among reaction norms was pronounced only within certain ranges of the environmental gradients. On the light and moisture gradients, genotypes were strikingly similar in their performance in all but the darkest and driest growing conditions (Fig. 2a, e). In contrast, maximum divergence of genotypic reaction norms occurred at the highest nutrient addition levels (Fig. 2c). Similar results of increased variation in suboptimal conditions have been reported, for example, from Polygonum (Sultan \& Bazzaz, 1993) and Taraxacum (Cox \& Ford, 1987). Overall similarity of ecological responses was very high among

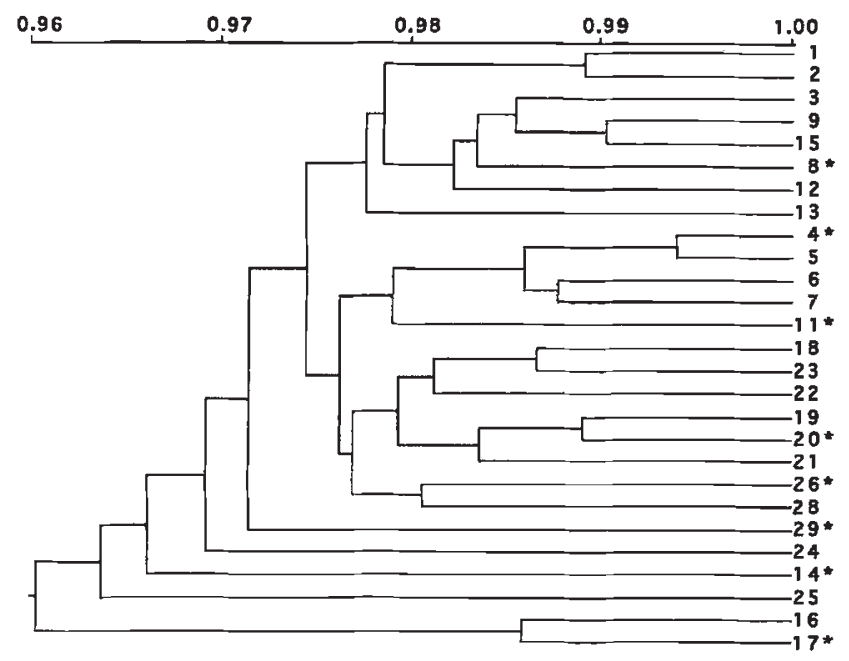

Fig. 1 Dendrogram of genetic similarities among 27 genotypes of Abutilon theophrasti, based on over 400 RAPD loci, with the cophenetic correlation coefficient of 0.855 $(P<0.001)$. Asterisks indicate genotypes used in the analyses presented in Figs 2 and 3. 
Fig. 2 Left panels: Norms of reaction of eight genotypes of Abutilon theophrasti tested along each environmental gradient (abscissa: values of environmental gradients at which experiments were conducted; ordinate: average of log-transformed total fruit biomass per plant in $\mathrm{mg}$ ). Right panels: Relationships between genetic similarities derived from RAPD analysis and phenotypic similarities as measured by Horn's overlap index. $P$-value corresponds to the significance of the linear regression obtained in Mantel's test. $(\mathrm{a}, \mathrm{b})$ light gradient; (c, d) nutrient gradient; $(e, f)$ moisture gradient; $(\mathrm{g}, \mathrm{h})$ temperature gradient.
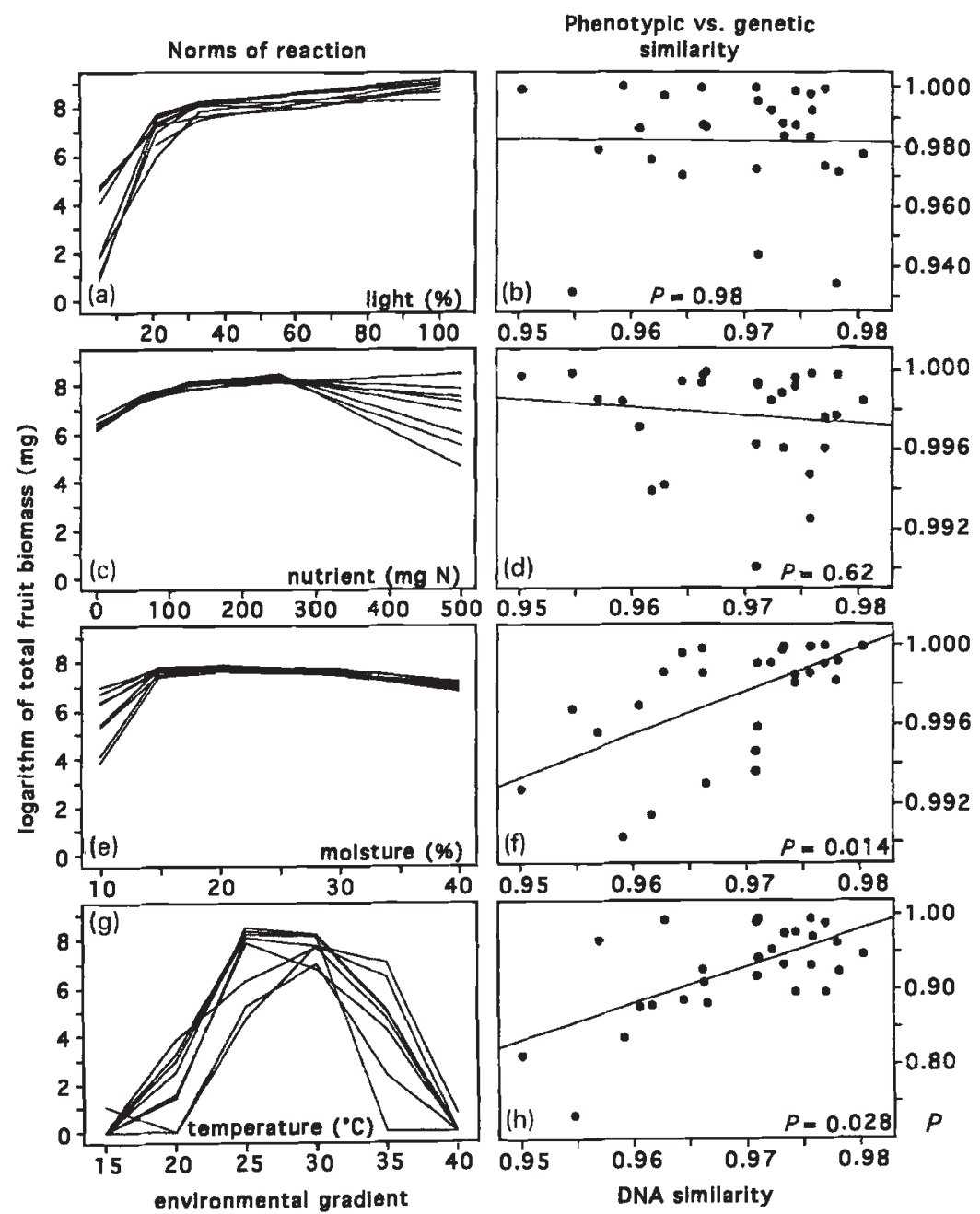

the eight focal genotypes, especially in the case of the nutrient and moisture gradients (Fig. $3 b, c$ ). Responses of genotypes to the temperature gradient were more varied, with subsets of genotypes differing in their optimum temperatures (Fig. 2g). Consequently, the index of phenotypic similarity varied more in the case of temperature than in other gradients (Figs $3 \mathrm{~d}$ and $2 \mathrm{~h}$ ). More detailed analyses of the growth responses of all genotypes on all four gradients and comparisons of the phenotypic similarity matrices across gradients will be presented elsewhere.

A remarkable result of this study is that, within the observed narrow range of genetic differences, on two gradients the index of similarity in total fruit biomass covaried significantly with genetic similarity. The slope of the linear regression of the index of phenotypic similarity on the index of DNA similarity was significantly greater than 0 in the case of the temperature $\left(r^{2}=0.41, P=0.028\right)$ and moisture gradients $\left(r^{2}=0.37, P=0.014\right)$, suggesting a genetic basis for phenotypic plasticity. However, there was no relationship between genetic and phenotypic similarity in the case of the light $\left(r^{2}=0, P=0.98\right)$ or nutrient gradients $\left(r^{2}=0.02, P=0.62\right)$. These results are confirmed in Mantel's tests performed on all genotypes tested on each of the gradients. There was a significant linear regression of phenotypic similarity on DNA similarity in the case of the temperature gradient $\left(r^{2}=0.13, P=0.039\right)$, and a marginally significant regression slope in the case of the moisture gradient $\left(r^{2}=0.07, P=0.058\right)$. Genetic and phenotypic similarity were not correlated in the case of the light $\left(r^{2}=0.01, P=0.54\right)$ or nutrient gradients $\left(r^{2}=0.02, \quad P=0.28\right)$. Why is the relationship between genotypic and phenotypic space different between moisture/temperature and light/nutrient gradients?

The absence of a relationship between genetic and phenotypic similarity could indicate weaker genetic 
(a)

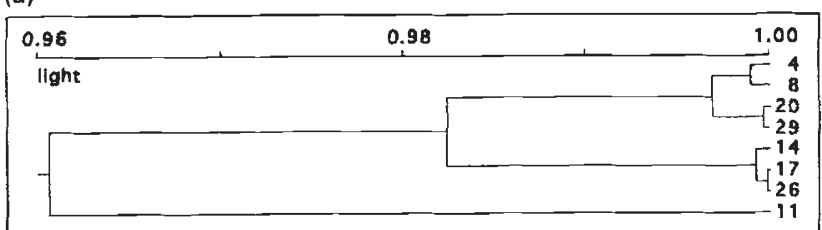

(b)

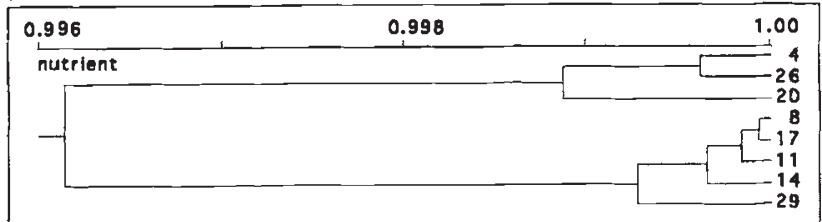

(c)

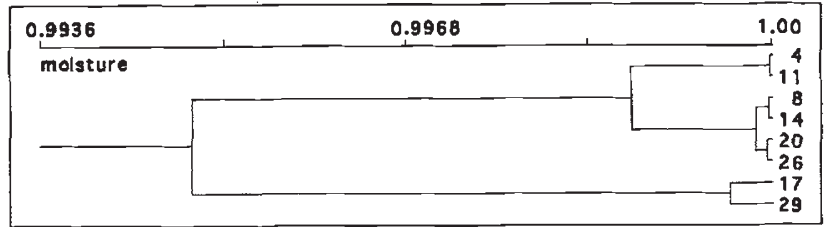

(d)

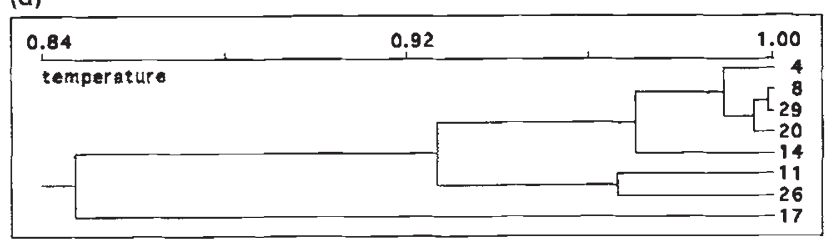

Fig. 3 Dendrograms of phenotypic similarities among eight genotypes of Abutilon theophrasti, which were grown on the single gradient of light (a), nutrient (b), moisture (c) and temperature (d). The cophenetic correlation coefficients were $0.766,0.795,0.675$ and 0.884 , respectively (all significant at $P<0.001$ ).

underpinnings than in the case of the moisture and temperature gradients (see Pigliucci et al., 1995). Conversely, plasticity with respect to variation in light or nutrients could be caused by a relatively small number of major-effect loci or regulatory elements (see Schlichting \& Pigliucci, 1995; Via et al., 1995). Adaptations based on major-effect loci seem, in fact, to be more widespread than the 'micromutationist' view has previously allowed (Orr \& Coyne, 1992; Mitchell-Olds, 1995). The methods frequently have little power of detecting major-effect loci, however, and the conclusive evidence requires more than a correlational approach presented here. Environmental sensitivity of loci, which are members of sets of functionally interrelated genes, can have similar expression to single loci of major effects (Moreno, 1994). Moreover, there is evidence that the presence and the magnitude of effects of quantitative trait loci (QTL) may vary between environments (Tanksley, 1993). Under such a scenario of environment-QTL interaction, one may expect some instances of environmental conditions associated with pronounced major loci effects (for example, corresponding to the light and nutrient gradient in this study), and other instances of environments with more uniform distributions of gene effects (corresponding to moisture and temperature gradients in this study).

The approach advocated here does not yield specific information about the identity of loci contributing to the phenotypic variation. A more advanced analysis correlating phenotypic and molecular variation in the region causally involved in influencing the trait in question has been developed (see e.g. Lai et al., 1994). Our approach may nevertheless provide rapid insight into the extent to which genetic variation underlies the phenotypic properties of organisms (Nei, 1987; Orr \& Coyne, 1992). The method could be especially valuable in studies of long-lived organisms in which quantitative genetic methods are not feasible, or of species with small genomes, for which a thorough RAPD analysis can yield reliable estimates of the overall DNA similarity. The requirement of intense sampling of the genome is especially critical, because conclusions from correlational analyses that are based on a lack of a relationship between phenotypic and genotypic space must be protected against type II error (accepting a hypothesis of no relationship, whereas in fact a congruence exists). Although the number of bands used in this study for estimating genomic similarity (on average over 400) is quite high according to current standards, we believe that satisfactory sampling of genome similarity via RAPD analysis in higher organisms may require thousands of bands. The resulting estimate of genetic similarity may be, however, more reliable than that derived from pedigree data alone (Tinker et al., 1993).

An insight into the genetic basis of plasticity is important, as plasticity influenced by major-effect loci is likely to have different evolutionary dynamics (e.g. short-term response to selection) than if it were controlled by many loci with minor effects (Lande, 1983; Orr \& Coyne, 1992; Mitchell-Olds, 1995). Furthermore, an awareness of the extreme context dependence of the genetic underpinnings of plasticity is critical. Potential evolutionary pressures resulting, for example, from variation in light intensity or soil nutrients, may be qualitatively different from those caused by variation in soil moisture (Pigliucci et al., 1995). Our results point, therefore, to an enormous complexity of selective pressures and selective responses among plants growing under simultaneous and interacting spatial and temporal heterogeneity of conditions. 


\section{Acknowledgements}

We thank D. Buckley and K. Garbutt for their participation in the ecological part of the project and $\mathrm{D}$. Ackerly, G. Berntson, D. Hartl, L. Hughes, R. Lewontin, S. Sultan and M. Westoby for helpful discussions. We also thank D. Hartl for providing the facilities for the RAPD analyses (supported by the NIH grant GM14846 to F.J.A.). This study was supported by the National Science Foundation grant BSR-8414355 to F.A.B.

\section{References}

Bachmann, K. AND VAn heusen, A. w. 1992. Phenotypic similarity and genetic relationship in Microseris elegans (Asteraceae: Lactuceae). Biol. Zentralbl., 111, 38-53.

BAzZAZ, F. A. 1996. Plants in Changing Environments. Cambridge University Press, Cambridge.

BAZZAZ, F. A., JASIEŃSKI, M., THOMAS, S. C. AND WAYNE, P. 1995. Microevolutionary responses in experimental populations of plants to $\mathrm{CO}_{2}$-enriched environments: parallel results from two model systems. Proc. Natl. Acad. Sci. U.S.A., 92, 8161-8165.

COUNTS, R. L. 1993. Phenotypic plasticity and genetic variability in annual Zizania spp. along a latitudinal gradient. Can. J. Bot., 71, 145-154.

COX, T. AND FORD, H. 1987. The plastic growth responses of three agamospecies of dandelion to two levels of nutrient. Ann. Bot., 59, 81-91.

DAMERVAL, C., HÉBERT, Y. AND VIENNE, D. D. 1987. Is the polymorphism of protein amounts related to phenotypic variability? A comparison of two-dimensional electrophoresis data with morphological traits in maize. Theor. Appl. Genet., 74, 194-202.

HADRYS, H., BALICK, M. AND SCHIERWATER, B. 1992. Applications of random amplified polymorphic DNA (RAPD) in molecular biology. Mol. Ecol., 1, 55-63.

KREBS, C. J. 1989. Ecological Methodology. Harper \& Row, New York.

LAl, C., LYMAN, R. F., LONG, A. D., LANGLEY, C. H. AND MACKAY, T. F. C. 1994. Naturally occurring variation in bristle number and DNA polymorphisms at the scabrous locus of Drosophila melanogaster. Science, 266, 1697-1702.

LANDE, R. 1983. The response to selection on major and minor mutations affecting a metrical trait. Heredity, 50, 47-65.

LANDER, E. S. AND SCHORK, N. J. 1994. Genetic dissection of complex traits. Science, 265, 2037-2048.

LEWONTIN, R. C. 1974. The Genetic Basis of Evolutionary Change. Columbia University Press, New York.
MACDONAld, S. E. AND ChInNaPPa, c. c. 1989. Population differentiation for phenotypic plasticity in the Stellaria longipes complex. Am. J. Bot., 76, 1627-1637.

manly, B. F. J. 1991. Randomization and Monte Carlo Methods in Biology. Chapman \& Hall, London.

MITCHELL-OLDS, T. 1995. The molecular basis of quantitative genetic variation in natural populations. Trends Ecol. Evol., 10, 324-328.

MORENO, G. 1994. Genetic architecture, genetic behaviour, and character evolution. Ann. Rev. Ecol. Syst., 25, $31-44$.

NEl, M. 1987. Molecular Evolutionary Genetics. Columbia University Press, New York.

ORR, H. A. AND COYNE, J. A. 1992. The genetics of adaptation: a reassessment. Am. Nat., 140, 725-742.

PigliuCCI, M., whitTon, J. AND schlichting, C. D. 1995. Reaction norms of Arabidopsis. 1. Plasticity of characters and correlations across water, nutrient and light gradients. J. Evol. Biol., 8, 421-438.

ROHLF, F. J. 1994. NTSYS-pc. Numerical Taxonomy and Multivariate Analysis System. Version 1.80. Exeter Software, Setauket, NY.

SCHARLOO, w. 1991. Canalization: genetic and developmental aspects. Ann. Rev. Ecol. Syst., 22, 65-94.

SCHEINER, S. M. 1993. Genetics and evolution of phenotypic plasticity. Ann. Rev. Ecol. Syst., 24, 35-68.

schlichting, C. D. AND LEvin, D. A. 1984. Phenotypic plasticity of annual Phlox: tests of some hypotheses. Am. J. Bot., 71, 252-260.

SCHLichting, C. D. AND PIGLiUCCl, M. 1995. Gene regulation, quantitative genetics, and the evolution of reaction norms. Evol. Ecol., 9, 154-168.

SUltan, s. E. AND BAzZaz, F. A. 1993. Phenotypic plasticity in Polygonum persicaria. I. Diversity and uniformity in genotypic norm of reaction to light. Evolution, 47, 1009-1031.

TANksley, S. D. 1993. Mapping polygenes. Ann. Rev. Genet., 27, 205-233.

TAYLOR, D. R. AND AARSSEN, L. w. 1988. An interpretation of phenotypic plasticity in Agropyron repens (Graminae). Am. J. Bot., 75, 401-413.

TINKER, N. A., FORTIN, M. G. AND MATHER, D. E. 1993. Random amplified polymorphic DNA and pedigree relationships in spring barley. Theor. Appl. Genet., 85, 976-984.

VIA, S., GOMULKIEWICZ, R., DE JONG, G., SCHEINER, S. M., SCHLICHTING, C. D. AND VAN TIENDEREN, P. 1995. Adaptive phenotypic plasticity: consensus and controversy. Trends Ecol. Evol., 10, 212-217.

VILlANI, F., PIGLIUCCI, M., LAUTERI, M., CHERUBiNI, M. AND sun, O. 1992. Congruence between genetic, morphometric, and physiological data on differentiation of Turkish chestnut (Castanea sativa). Genome, 35, 251-256. 